\title{
La pérennisation des documents \\ numériques
}

Quelles solutions pour

> Le développement du numérique dans tous les secteurs d'activité entraîne une production croissante de documents numériques qu'il est impératif de conserver durablement. Le secteur les petites structures?

\section{Claude Huc} de la santé n'y échappe pas. La préservation à long terme de ces documents se heurte à la faible pérennité des technologies et des supports de stockage. Un certain nombre de grandes industries et d'organismes nationaux ont mesuré les risques et mettent en place des moyens et des équipes pour faire face à ce défi. À l'inverse, les petites structures, cabinets médicaux, individus isolés, étudiants, etc. n’ont généralement pas conscience du problème ou sont démunis pour y faire face. Un certain nombre d'actions simples, n'exigeant pas de compétences spécifiques en informatique, peuvent néanmoins être entreprises. Sans dépenses importantes, elles augmentent de façon significative le niveau de sécurité des documents à long terme. <



Consultant indépendant, 3 , chemin du Couderla, 31320 Vigoulet-Auzil, France. claude.huc@free.fr

simple de certains documents nécessaires à la bonne marche de nos activités.

De grands organismes ont pris conscience du problème et mettent en œuvre des moyens lourds et des compétences expertes pour assurer la préservation des documents. Dans le domaine de la recherche, la prise de conscience émerge de façon inégale au sein des plus grands instituts. II n'en est pas de même pour les groupes de taille modeste, les unités de recherche les plus petites, les cabinets libéraux, les doctorants, les chercheurs plus isolés ou encore les associations qui interviennent dans le domaine des questions de santé.

Le numérique envahit progressivement tous les domaines d'activités professionnelles et personnelles. C'est un rouleau compresseur qui élimine et entraîne la mort de nombre de technologies qui le précédaient. Qu'il s'agisse de photographie, d'imagerie médicale, d'instrumentation scientifique, de plans, de dessins, etc., les méthodes ou techniques traditionnelles cèdent la place au numérique qui permet d'aller plus vite, de faire mieux avec des coûts moindres.

Ce mouvement aboutit à une production croissante de documents numériques que nous avons besoin de conserver durablement pour de multiples raisons: impératifs scientifiques, contraintes légales, précautions juridiques, nécessités patrimoniales, choix personnels, etc. Malheureusement, la puissance extraordinaire du numérique se heurte à la courte durée de vie des technologies matérielles et logicielles sur lesquelles elle s'appuie (Figure 1). Nous n'avons pas encore tous mesuré le risque majeur que pose le numérique à un horizon de dix ans ou plus, à savoir la perte pure et pas les réponses. Hormis un premier guide pratique [1], il n'existe pratiquement pas de travaux sur ces cas de figure. Le rapport du groupe de travail mandaté par l'Académie des sciences et l'Académie des technologies avait souligné à quel point le problème de conservation des documents numériques à long terme « est très largement ignoré du grand public ainsi que de la majorité des institutions ou entreprises » [2].

Après avoir examiné le contexte général et celui de la recherche scientifique, c'est à la gestion du problème par les petites structures que cette revue s'intéresse de façon plus approfondie.

\section{Un changement spectaculaire du contexte depuis dix ans}

Depuis dix ans, nombre de grandes entreprises et d'organismes nationaux ont pris la mesure des risques, des 


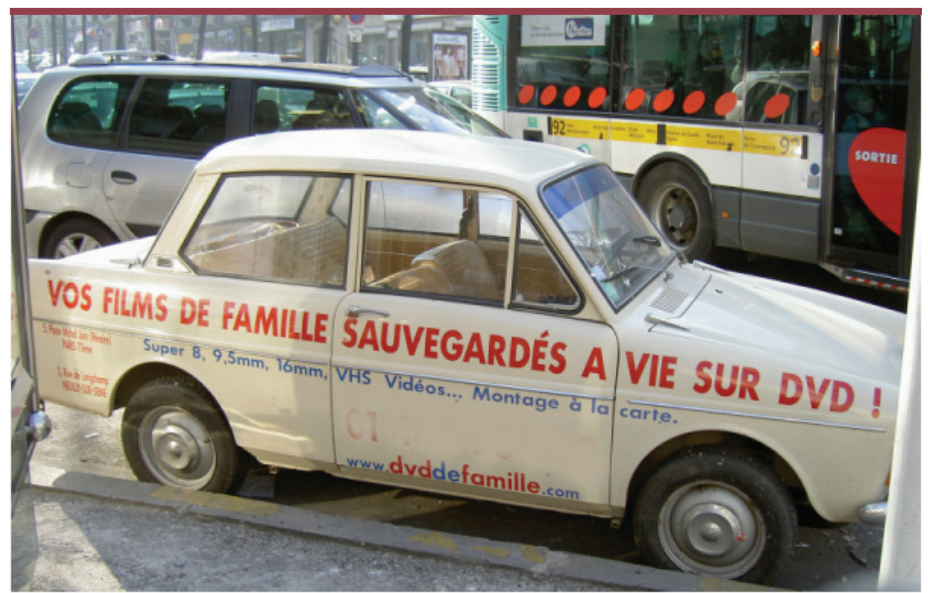

Figure 1. Les idées reçues sont parfois véhiculées de façon surprenante (photo : (c) Jean-Marc Fontaine).

enjeux et ont mis en place des équipes et des moyens pour garantir la pérennité de leurs documents numériques. Un exemple frappant est celui de l'industrie aéronautique. La documentation, entièrement numérique, d'un avion de ligne contient la description de chacune des pièces constituant cet avion. La durée de vie de l'avion et les contraintes juridiques impliquent une conservation de cette documentation pendant plus de cinquante ans. En 2008, Airbus et Boeing, par ailleurs féroces concurrents, ont signé, par l'intermédiaire des associations aérospatiales américaines et européennes, un mémorandum d'accord sur l'archivage à long terme des données qu'ils produisent. Cet accord a abouti à la création du projet LOTAR (long term archiving and retrieval [3]) qui vise à apporter des solutions aux problèmes de pérennisation des documents produits par ces industries.

Dans le domaine patrimonial, si l'Institut national de l'audiovisuel (Ina) n'avait pas mis en place son projet «plan de sauvegarde et de numérisation », 835000 heures d'archives auraient disparu d'ici 2015, soit près d'un tiers des archives audiovisuelles conservées par l'Ina [4]. De son côté, la Bibliothèque nationale de France $(\mathrm{BnF})$ a mis en service en 2010 son très ambitieux système de préservation et d'archivage réparti (SPAR) [5]. Ce système constitue aujourd'hui une réalisation en tous points conforme aux normes essentielles du domaine et en particulier à la norme ISO 14721 «Modèle de référence pour un système ouvert d'archivage d'information » $[6,7]$. Enfin, le Service interministériel des archives de France (SIAF) [8] mène actuellement une politique active visant à rationaliser l'activité de préservation numérique dans les différents ministères.

De leur côté, les établissements hospitaliers mènent de front plusieurs opérations d'envergure :

- la transition vers un dossier médical entièrement numérique. À I'AP-HP (Assistance publique - Hôpitaux de Paris), I'imagerie était presque totalement dématérialisée fin 2010. Une dématérialisation totale, incluant résultats d'analyses, comptes-rendus des médecins, etc., est planifiée pour 2015 ;

- une réorganisation du dispositif informatique impliquant une forte réduction du nombre des applications logicielles utilisées;
- la mise en place de procédures de sauvegarde sécurisée avec un stockage des données sur plusieurs sites géographiquement distants en attendant la conduite d'une réflexion de fond sur la problématique «long terme ». À cet égard, les établissements de santé rencontrent une diversité et une complexité de documents tout aussi grande que celle des autres grands organismes.

Cette problématique se pose dans les mêmes termes pour le dossier médical personnel (DMP) [9] ou pour les agences sanitaires [10].

Un partage des approches et des retours d'expérience en matière de préservation à long terme des documents numériques est organisé depuis plus de dix ans au sein du groupe PIN (pérennisation des informations numériques) [11]. La conservation de l'information numérique avait fait l'objet d'une analyse dans médecine/sciences en 2008 [12] et d'un développement beaucoup plus complet sous la forme d'un ouvrage destiné aux acteurs principaux de cette conservation en 2009 [13].

\section{Le monde de la recherche scientifique cherche sa voie}

Le Centre national d'études spatiales (CNES) et le Centre national de la recherche scientifique (CNRS) ont signé ensemble des protocoles d'accord visant à créer des centres de données en charge de la pérennisation des observations issues des expériences scientifiques embarquées. Le Centre de données de la physique des plasmas (CDPP) [14] en est un exemple.

Le Centre informatique de l'enseignement supérieur (CINES) s'est vu confier une mission nationale d'archivage pérenne de documents numériques du patrimoine scientifique [15]. La mission du CINES pour l'archivage des thèses numériques a fait l'objet d'un arrêté au Journal officiel le 7 août 2006. Le CINES archive un certain nombre de sources de documents numériques parmi lesquelles les publications du portail HAL (hyper article en ligne) des archives ouvertes [16, 20], et médecine/ sciences y avait consacré un article [21].

L'une des initiatives du TGE (très grand équipement) Adonis ${ }^{1}$ du CNRS [17] vise à assurer la préservation des données numériques issues de la recherche dans le domaine des sciences humaines et sociales en se basant sur une infrastructure d'archivage mutualisée entre le CINES et le Centre de calcul de l'Institut national de physique nucléaire et de physique des particules (CC-IN2P3). Ces initiatives pilotes ne couvrent qu'une partie réduite de la production scientifique française. L'archivage

${ }^{1}$ Adonis : accès unifié aux données et documents numériques des sciences humaines et sociales. http://www.tge-adonis.fr 
Kodak CD-R Preservation Gold 5 pack (580138)



Figure 2. Quelques exemples de publicités trouvées sur internet. Elles annoncent avec sérieux des durées de vie des CD de 100 et 300 ans!

numérique de la production des chercheurs se heurte à la multiplication des unités et des équipes de recherche et à la grande autonomie des chercheurs. L'Institut national de la santé et de la recherche médicale (Inserm) a entrepris en 2009 une réflexion sur la mise en place d'un système d'archivage numérique. La situation n'a cependant guère avancé depuis.

\section{Les besoins des structures les plus petites et des individus isolés}

Les structures de taille réduite et a fortiori les individus isolés ne disposent ni des moyens ni des compétences pour appréhender le problème de la même façon que les grands organismes. Pourtant, chacun, au sein de son équipe ou de son activité professionnelle individuelle, a besoin de conserver un certain nombre de documents : rapports et mémoires, études, publications, etc. À ces documents s'ajoutent ceux qui relèvent d'obligations légales. Pour les médecins libéraux, ce sera par exemple le dossier de suivi médical que le Conseil de l'Ordre recommande de conserver vingt ans, certains courriers, la comptabilité, les documents fiscaux, etc. Dans quelques cas, ces petites structures peuvent s'adosser aux systèmes mis en place par de plus grands organismes comme la BnF ou le CINES qui jouent alors le rôle de tiers archiveur. Cependant, elles sont très nombreuses à ne pas avoir cette possibilité. Comment alors assurer la conservation des documents avec un niveau de sécurité suffisant et sans dépenses significatives?

Les particuliers, dans leur vie privée, se posent la même question. Ce sont les photographies numériques qui ont déclenché la constitution d'un patrimoine numérique personnel. À cela s'ajoutent les vidéos, les courriers importants, les documents issus de nos passions, généalogie, musique, etc., c'est-à-dire tout ce qui constitue un patrimoine affectif ou artistique auquel s'ajoutent les documents numériques qui seront utiles pour faire valoir nos droits : factures, relevés de banque, relevés de Sécurité sociale, déclarations fiscales.
Le risque de tout perdre et quelques solutions pragmatiques pour l'éviter

Les dangers de tout perdre sont multiples et partiellement méconnus. Le vol de l'ordinateur et du disque dur de sauvegarde posé juste à côté, le sinistre, l'infection par un virus destructeur ne sont pas si rares. Mais, avec quelques précautions, il est possible de réduire les risques. Une situation plus insidieuse conduira à l'illisibilité de certains documents du fait de l'obsolescence des formats de fichiers ou de la dégradation des supports d'archivage les renfermant. Conscient de ces risques, chacun sera donc peu à peu conduit à appliquer d'une manière ou d'une autre un ensemble des recommandations présentées ci-après. Bien que fort limitées et peu détaillées, les actions proposées ci-après sont de nature à apporter une relative sécurité des documents numériques à conserver au cours du temps.

\section{Savoir ce que l'on veut préserver}

C'est la première des actions à mener. Elle n'exige aucune connaissance technique particulière. La conservation de ce que nous appelons le patrimoine numérique ne pourra être convenablement assurée que si nous avons précisément identifié les documents à préserver. On conservera ce qui aura une valeur ou une utilité dans le futur. La préservation à long terme ne concerne donc ni les multiples documents d'usage éphémère ni les versions intermédiaires de travail.

\section{Regrouper et nommer soigneusement \\ ce qui doit être préservé}

Le disque dur de l'ordinateur ressemble à une gigantesque bibliothèque dans laquelle nous pourrions ranger des dizaines de milliers de documents. Si nous ne prenons pas la peine de regrouper au sein d'une organisation bien structurée de dossiers tout ce qui nous paraît devoir être pérennisé, nous aurons toutes les chances de ne plus nous y retrouver dans le futur. La dispersion des documents sur le disque dur est en partie provoquée par les logiciels qui suggèrent d'enregistrer les documents dans les dossiers les plus variés. Cette dispersion peut aussi être aggravée par la négligence de l'utilisateur. Une fois les documents regroupés, il convient de leur donner des noms explicites susceptibles de traverser le temps.

\section{Savoir à quoi s'en tenir} sur les supports de stockage

En excluant les technologies réservées aux grands sites informatiques, nous avons le choix entre trois catégo- 


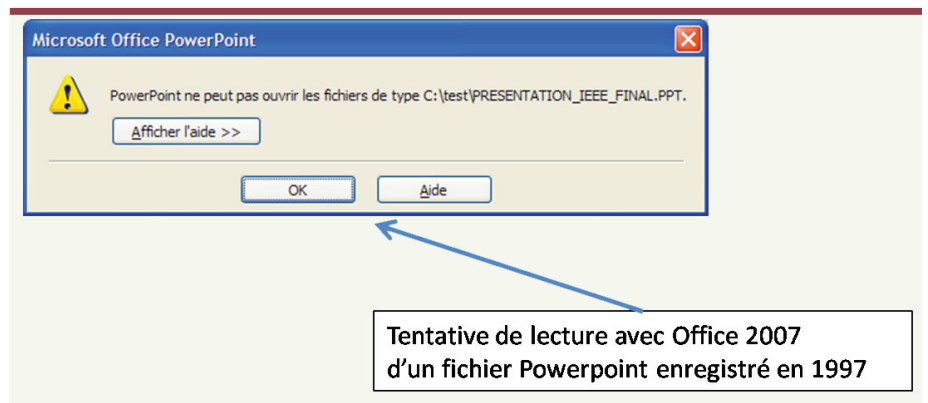

Figure 3. Les logiciels de la suite Microsoft Office 2007 ou 2010 ne savent plus reconnaître les fichiers créés 10 ou 12 ans plus tôt par Word ou PowerPoint de Microsoft !

ries: le disque dur, les disques optiques (CD, DVD, Blu-Ray ${ }^{2}$ ) et les mémoires flash (clés USB, cartes mémoires des appareils photos, etc.). Le recul sur la durée de vie des mémoires flash est aujourd'hui insuffisant. La durée de vie des disques durs ne dépasse guère cinq ans en moyenne et le disque peut cesser de fonctionner sans préavis. Le dysfonctionnement du disque dur constitue d'ailleurs la première cause de panne des ordinateurs. Pour les disques optiques, les informations les plus contradictoires circulent. Ces informations sont polluées par le discours marketing d'un certain nombre de vendeurs (Figure 2).

Nous devons nous en tenir aux travaux du Laboratoire national d'essais et de métrologie (LNE), laboratoire public, neutre et indépendant de tout intérêt marchand. Le LNE a montré, sur la base d'analyses statistiques représentatives sur des CD et des DVD entreposés sur des sites différents, que la durée de vie de ces disques est souvent réduite à deux, trois ou cinq ans. Tous les disques ne sont pas pour autant équivalents mais il n'est pas facile de connaître a priori ceux qui sont plutôt fiables et ceux qui ne le sont pas. Ces analyses sont disponibles en ligne sur le site du pôle de recherche sur la conservation des données sur disques optiques numériques [18].

\section{À propos des services de stockage en ligne}

Ces services sont appelés à jouer un rôle important dans le futur. Ils permettent indirectement l'usage de moyens de stockage lourds et sécurisés. Cependant, la situation n'est pas encore mûre. Les services de stockage gratuits sont à exclure totalement. La plupart d'entre eux se réservent le droit d'utiliser nos données stockées. Les services de stockage payants sont à utiliser avec beaucoup de prudence. Lorsqu'on ne connaît pas la localisation du site de stockage, on peut s'interroger sur les garanties de confidentialité. En outre, en fonction des services, on observe aussi des écarts de prix extravagants.

${ }^{2} \mathrm{CD}$ : compact disc ; DVD : digital versatile disc ; « le disque Blu-ray ou Blu-ray disc (abréviation officielle $B D$, autre dénomination $B-R D$ ) est un format de disque numérique breveté et commercialisé par l'industriel japonais Sony permettant de stocker et restituer des vidéogrammes en haute définition. Sa dénomination provient du type de rayon laser qu'il exploite, de couleur spectrale proche du bleu » (Wikipédia).
Choisir les supports adaptés, disposer de plusieurs copies et les surveiller

La seule réponse crédible face à ces constats consiste à réaliser plusieurs copies des mêmes documents et d'en assurer régulièrement la surveillance et le renouvellement. Le choix des types de support dépend du volume de données à stocker. Pour stocker 500 Go de données et de documents, il sera préférable de choisir un disque dur plutôt qu'une centaine de DVD et $a$ fortiori un millier de CD. Un nombre réduit de supports de stockage facilitera leur surveillance. En l'absence d'information fiable sur le vieillissement des supports, cette surveillance consiste à effectuer, au moins annuellement, une relecture complète de l'ensemble des copies. On pourra par exemple recopier la totalité des fichiers sur un espace tampon. Si l'un des supports est défaillant, il devra alors être remplacé par un support neuf reconstitué à l'aide des copies valides. Une bonne méthode consiste à gérer un tableau de bord des supports et des opérations réalisées comme le montre l'exemple du Tableau I.

Deux exemplaires du patrimoine numérique ne suffisent pas : si au moment de la défaillance du disque dur de l'ordinateur, le disque dur de secours tombe, tout est perdu. Trois exemplaires peuvent suffire à condition de renouveler préventivement et régulièrement les supports, opération d'autant plus difficile que nous n'avons pas à notre disposition de moyens fiables de prédiction. C'est pourquoi nous préconisons quatre exemplaires dont l'un doit être impérativement stocké dans un lieu géographique distinct des autres.

\section{Les documents sans logiciel de lecture}

Pour de multiples raisons, un éditeur de logiciel peut disparaître du jour au lendemain. Les logiciels qu'il distribue ne seront alors plus mis à jour ni adaptés aux nouveaux systèmes d'exploitation. Tout aussi préjudiciable: certains logiciels mondialement utilisés, comme la suite Microsoft Office, n'assurent que partiellement la compatibilité ascendante permettant de lire les anciens fichiers avec les nouvelles versions de ces logiciels (Figure 3).

Pour les documents très courants, nous pourrons cependant avoir une relative confiance dans les formats PNG, JPEG, JPEG2000 pour les images et dans les versions normalisées de PDF : PDF/A (ISO 19005-1) et PDF 1.7 (IS0 32000-1). Pour les documents spécifiques à chaque discipline, on examinera la situation au cas par cas avec plusieurs principes : exclure les formats non publiés, privilégier les formats ouverts et minimiser le nombre de formats différents utilisés. 


\begin{tabular}{|c|c|c|c|c|c|}
\hline Date & Action & Version de référence & Copie 1 & Copie 2 & Copie 3 \\
\hline $21-10-2007$ & $\begin{array}{l}\text { Création version } \\
\text { initiale }\end{array}$ & $\begin{array}{c}\text { Disque dur } 500 \text { Go My book } \\
\text { de Western Digital } \\
\text { Création et relecture OK } \\
12143 \text { fichiers } \\
211 \text { Go }\end{array}$ & & & \\
\hline $22-10-2007$ & COPIE & & $\begin{array}{c}\text { Disque dur } \\
\text { lomega Prestige Desk- } \\
\text { top hard drive } 500 \text { Go } \\
\text { OK }\end{array}$ & $\begin{array}{l}\text { Disque dur } \\
\text { Intenso memory } \\
\text { house } 320 \text { Go } \\
\text { OK }\end{array}$ & $\begin{array}{l}\text { Disque dur } \\
\text { Intenso memory } \\
\text { house } 320 \text { Go } \\
\text { OK }\end{array}$ \\
\hline $15-04-2008$ & $\begin{array}{l}\text { Vérification: } \\
\text { relecture totale }\end{array}$ & OK & OK & OK & OK \\
\hline $12-07-2008$ & $\begin{array}{l}\text { Mise à jour version } \\
\text { de référence avec } \\
\text { nouveaux documents }\end{array}$ & $\begin{array}{c}13665 \text { fichiers } \\
243 \text { Go }\end{array}$ & & & \\
\hline $13-07-2008$ & Mise à jour copies & & OK & OK & OK \\
\hline $09-12-2008$ & Vérification & OK & OK & OK & OK \\
\hline Etc. & & & & & \\
\hline $22-10-2010$ & Vérification & OK & OK & $\begin{array}{l}\text { Disque illisible - } \\
\text { remplacement du } \\
\text { disque dur Western } \\
\text { Digital Elements } \\
\text { (1 To) }\end{array}$ & OK \\
\hline
\end{tabular}

Tableau I. Un exemple de ce que peut être un tableau de bord des supports de stockage.

\section{Au-delà de cette réflexion}

La société contemporaine a vu progressivement se développer le règne des objets éphémères. Rien de ce qui se fabrique aujourd'hui n'est construit pour durer. Pu'il s'agisse de l’ordinateur, du téléphone mobile ou du lave-linge, chaque appareil est conçu pour avoir une durée de vie limitée. Ce processus cyclique au cours duquel chacun acquiert, utilise, jette puis remplace ne concerne pas que les objets matériels. L'information - considérée de plus en plus souvent comme un produit comme un autre - subit les mêmes lois avec les mêmes résultats, probablement accentués par l'inflation vertigineuse de l'information disponible. Emmanuel Hoog, dans une analyse pertinente, qualifie le web de «fleuve d'amnésie » [19]. Cependant, et par opposition à ce processus comme pour tenter de le contrebalancer, les sociétés européennes et la société française tout particulièrement n'ont jamais été à ce point fébrilement attentives à leur passé et soucieuses de leur mémoire.

\section{Conclusions}

Nous n'avons fait que survoler la question et bien des aspects comme la préservation des courriels, celle des bases de données ou des sites web ou encore le sujet complexe des multiples formats vidéo n'ont pas été abordés. Les spécificités du secteur de la santé ne modifient pas les données du problème. Si le lecteur est quelque peu sensibilisé, cet article aura atteint son but. Ce lecteur pourra toujours recourir aux références pour chercher des solutions plus précises à ses besoins particuliers. $\diamond$

\section{SUMMARY}

Uncertainty of long term preservation of digital documents and how to cope with it 
The development of digital technologies in all activities sectors of our society leads to a growing number of digital documents. A significant part of these documents needs to be durably preserved. This long term preservation has to face the short life expectancy of the technologies and the digital storage media. Large national organizations have already take this problem into account and set up teams, skills and means to face this challenge. At the opposite, the small structures, doctor's offices, individuals, students, etc. are not generally aware of the problem or are stripped to face there. A certain number of simple actions, not requiring specific skills in data processing can nevertheless be undertaken. Without important expenditure, they increase to a significant degree, the security level of the documents over the long term. $\diamond$

\section{CONFLIT D'INTÉRÊTS}

L'auteur déclare n'avoir aucun conflit d'intérêts concernant les données publiées dans cet article.

\section{RÉFÉRENCES}

1. Huc C. Préserver son patrimoine numérique. Paris : Eyrolles, $2010: 324$ p.

2. Hourcade JC, Laloë F, Spitz $\varepsilon$. Longévité de l'information numérique. Paris : EDP Sciences, 2010 $106 \mathrm{p}$.

3. http://www.long-term-archiving-and-retrieval.org/

4. http://www.ina-entreprise.com/entreprise/activites/archives-sauvegarde-numerisation/index. html

5. http://www.bnf.fr/fr/professionnels/conserver_spar.html
6. Organisation internationale de normalisation ISO 14721-2003. Open archival information system - reference model. Genève : ISO 2003 : 164 p.

7. Organisation internationale de normalisation ISO 14721-2003. Système ouvert d'archivage d'information - modèle de référence. Genève : ISO 2003 $146 \mathrm{p}$.

8. http://www.archivesdefrance.culture.gouv.fr/annuaire-services/direction/

9. http://esante.gouv.fr/dmp

10. http://www.sante.fr/

11. http://pin.association-aristote.fr/doku.php

12. Huc $C$. La pérennisation des informations sous forme numérique : risques, enjeux et éléments de solution. Med Sci (Paris) 2008 ; 24 : 653-7.

13. Banat-Berger F, Duplouy L, Huc C. L'archivage numérique long terme : les débuts de la maturité ? Paris : La Documentation Française, 2009 : 284 p.

14. http://cdpp.cesr.fr/

15. http://www.cines.fr/spip.php?rubrique219

16. http://hal.archives-ouvertes.fr/

17. http://www.tge-adonis.fr/

18. http://www.Ine.fr/fr/r_et_d/gis-don/conservation-donnees-numeriquesgis-don.asp

19. Hoog E. Mémoire année Zéro. Paris : Seuil, 2009 : 208 p.

20. Duchange $N$, Autard $D$, Pinhas $N$. Le libre accès : une opportunité pour la recherche biomédicale. Med Sci (Paris) 2008 ; $24: 771-5$.

21. Rouchon 0 . La préservation de l'information scientifique et technique. PAC la plate-forme d'archivage pérenne de documents électroniques du Centre Informatique National de l'Enseignement Supérieur. Med Sci (Paris) 2008 ; 24 : 1099-102.

\section{Apprenez à respirer à vos enfants}

\section{Jean-Paul Allaux}

Les maladies ORL avec leur prolongement au niveau pulmonaire sont malheureusement de plus en plus répandues de nos jours et à tout âge.

La pollution atmosphérique, les appartements surchauffés ou enfumés par des parents tabagiques, les rues des villes saturées de gaz d'échappement... tous ces maux contemporains bien connus sont bien souvent responsables de l'installation de ces affections dès la petite enfance. Ils freinent par ailleurs le développement harmonieux du corps e $n$ installant parfois des déformations vertébrales mais aussi du palais et des mâchoires provoquant une mauvaise implantation des dents.

Fruit d'une longue expérience, ce livre se veut avant tout pratique. De nombreux exercices spécifiques à faire à la maison ou à l'école, seul ou en groupe, permettront aux éducateurs, parents et enseignants de trouver remède à ces différentes affections et d'aider efficacement les enfants à s'épanouir pleinement.

\section{TIRÉS À PART}

C. Huc

Un livre pour apprendre à respirer à nos enfants.



Commandez directement sur : www.edition-sciences.com 